\title{
Description of Two-Peak Characteristics in Power Engineering
}

\section{Tetiana Lutchyn}

National Technical University of Ukraine "Kyiv Polytechnic Institute", Institute for Energy Saving and Energy Management, Kyiv, Ukraine

\section{Email address:}

t.lutchyn@mail.ru

\section{To cite this article:}

Tetiana Lutchyn. Description of Two-Peak Characteristics in Power Engineering. American Journal of Environmental Protection. Vol. 4, No. 2, 2015, pp. 95-100. doi: 10.11648/j.ajep.20150402.14

\begin{abstract}
Many branches of Power Engineering have the same problems with signal processing. However they can be solved by a general approach. One of these problems is the complexity of signal description with the inertial change (without sharp peaks and dips), but the signal is a complex shape without symmetry and poorly responds to the cycling laws. Also offered, if it is necessary, to use the segmentation of the original signal with further Multiresolutional analysis. As a result, it is possible to make the selection with the most informative wavelet coefficients which can significantly reduce the quantity of the original data set with accuracy within acceptable limits.
\end{abstract}

Keywords: Multiresolutional Analysis, Wavelet Analysis, Description, Two-Peak Characteristics

\section{Introduction}

Nowadays there are a lot of methods, but it is very difficult to choose the unique algorithm for the data set automatically description. Especially the solution research is very difficult for asymmetrical signals with aperiodic character and additional conditions. Such type of signals could be with relative insignificant fluctuations, for example, two-peak characteristic and similar to them which can change with some inertness [1].

Description methods for such characteristics are: partial straightening [2], harmonious approach [3], two-level fuzzy models [4] and others. As a rule, signals of the specified character are observed in problems of: water supply [5], magnetic-electroencephalography [6], seismology [7, 8], climate change with taken into account a level of cloudy influence [9], value of precipitation [10], genetics [11], astronomy [12], topology [13] and other directions.

In power engineering, especially often similar signals are detected in tasks of:

- control of level of electricity generation [14];

- choice of electric equipment in distributive networks [15];

- installation of a network configuration [16];

- definition of peak consumption [17];

- calculation of the electricity cost [18];

- design and adjustment of Smart Grid [19];
- classification of consumers by their types [20,21];

- calculation of losses in the transmission line [22];

- studying of the consumer behaviour [23].

Description approaches are most developed for short-term forecasting of daily graphs. In such tasks, the most various models are:

- according to average differential characteristics [24, 25];

- iterative [26];

- neural network [27-30] and artificial neural network [31];

- ARIMA with the further signals clustering [32];

- wavelet transformation and fuzzy logic [33];

- genetically optimized cascade neural networks with Kohen's modified clustering [34];

- hybrid fuzzy-logical, however these models in some cases provide errors during description of the original data $[35,36]$.

In the theory of wavelet transformation for the graphs description, the partial signal representation is depicted in [7, 37], and also for air streams [38], seismology [39], medicine [40], meteorology [41], topography [13] and other.

This paper is organized as follows. For creation general algorithm in all next parts new properties and research results are presented. In part 2, main approaches of wavelet transformation and property of the Multiresolutional analysis (MRA) with developed flow scheme of wavelet coefficients are given. Part 3 discusses the description algorithm of the graph similar to two-peak characteristic with features of 
wavelet coefficients, while part 4 looks into details of data recovering with shifting in wavelet coefficients. In last part, some conclusions are presented.

\section{Main Approaches of Wavelet Transformation and Property of the Multiresolutional Analysis}

One of the most effective methods for the depiction shape of daily graphs is presented in [42, 43]. However it is noted by authors, this method has hyper sensibility to any changes in a signal. Thus, the variation between calculated and original values can achieve up to the triple divergence on nearly $50 \%$ of all research range.

In this paper, the main accent is directed on the model creation with a possibility of correction of extremely large deviation, in such way the sensitivity decrease to the distribution law of the original data. Minimal quantity of necessary values for recovery signal should be provided. For this purpose the initial signal is transformed MRA. Received
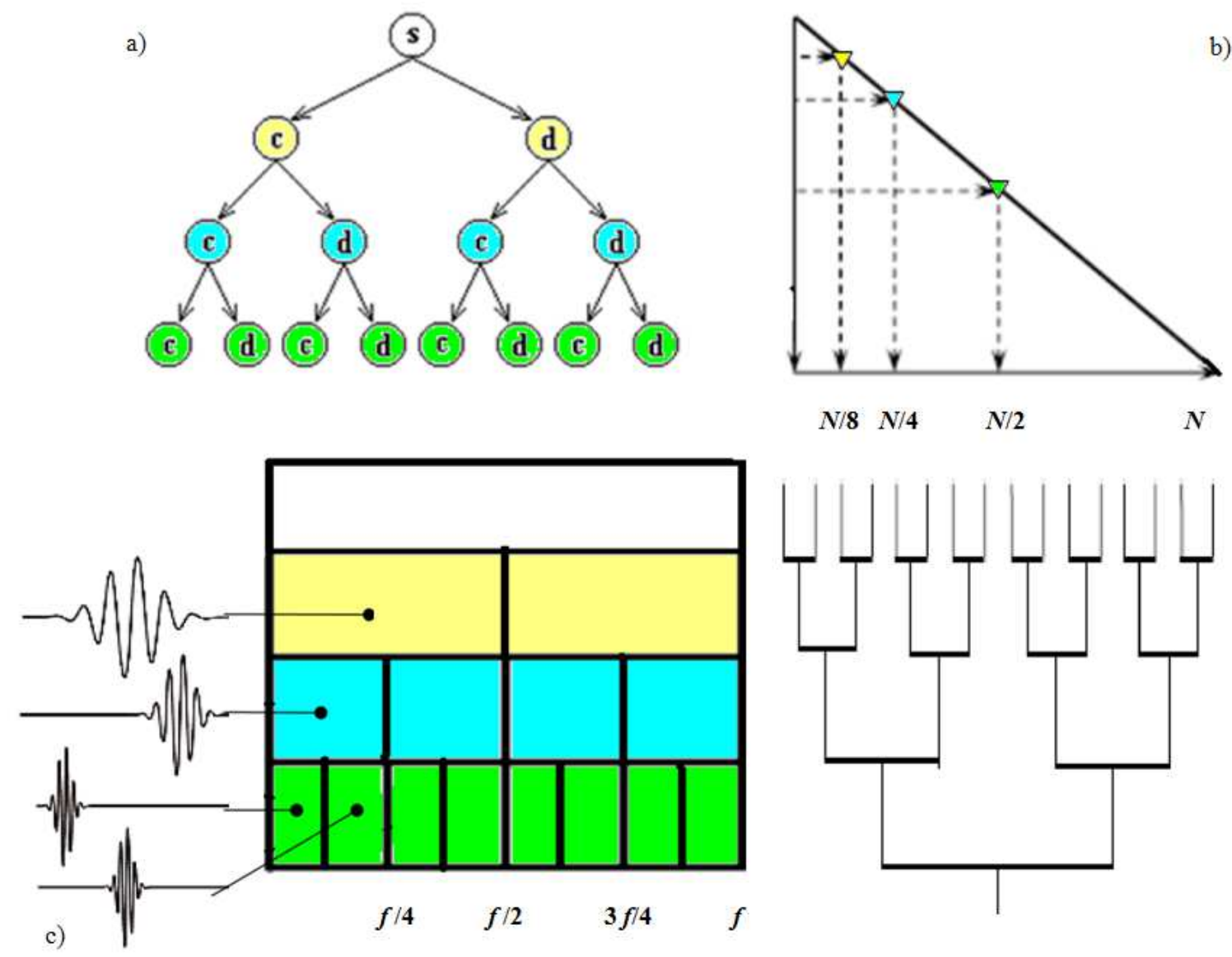

Fig. 1. Three-level decomposition tree of a signal with its main properties.

Let us present schematically a signal after MRA, as an example of three level decomposition tree

In fig. 1, a the most widespread scheme is presented in the wavelet theory. It is the basic principle of signal division according to the MRA main approach. Also for today's time wavelet coefficients are more informative, than the original signal without previous processing [42]:

$$
\begin{aligned}
& k_{m, 2 n}=\frac{k_{m-1,2 n}+k_{m-1,2 n+1}}{\sqrt{2}}, \\
& k_{m, 2 n+1}=\frac{k_{m-1,2 n}-k_{m-1,2 n+1}}{\sqrt{2}},
\end{aligned}
$$

where $k_{m, 2 n}$ and $k_{m, 2 n+1}$ - approximation and detailed wavelet coefficients, respectively; $m-$ level of transformation; $n$ - ordinal number of wavelet coefficient on the $m^{\text {th }}$ level of decomposition.

According to the inverse algorithm of MRA [42]

$$
\begin{aligned}
& k_{m-1,2 n}=\frac{k_{m, 2 n}+k_{m, 2 n+1}}{\sqrt{2}}, \\
& k_{m-1,2 n+1}=\frac{k_{m, 2 n}-k_{m, 2 n+1}}{\sqrt{2}},
\end{aligned}
$$

d)

the principles of its construction are well studied. The frequency division of a signal (it is one of the main properties of wavelet transformation) with its change are depicted in details in scale and in local representations (fig. 1, c).

In many papers with methods of data compression, authors 
very often miss the description of wavelet coefficients (fig. 1, b). However it is necessary to consider that on each level the calculated coefficients can contain different quantity of values. This problem is very important in the compression tasks for a correct choice of representative parameters for further data recovery. For example, that the original data set contains 16 values. According to the theory of wavelet decomposition, it provides four levels of transformation $\left(2^{m}=\right.$ $N, N$ - quantity of the initial values). There is a different quantity of wavelet coefficients with the different quantity of values on each level. For example, as a result of compression, it was defined that it was necessary to operate by two wavelet coefficients of the second level, one of the third level and two of the fourth level. It is incorrect to suppose that for full data recovery one can need $2+1+2=5$ values (these are wavelet coefficients!!!).

CONCLUSION 1: with increasing level of decomposition the quantity of wavelet coefficients increases in the same turn as the quantity of values decreases in wavelet coefficients.

This statement is confirmed also by fast wavelet decomposition by Mallat algorithm according to which one detailed wavelet coefficient of each level of decomposition and one approximate wavelet coefficient of the last wavelet level apply. For presented example it corresponds to:

$$
1 \times 8+1 \times 4+1 \times 2+2 \times 1=16 .
$$

In the classical theory of mathematics it is well known, that if quantity of required values equals to quantity of dissimilar equations, the task is completely defined and solutions are identified unambiguously.

As a research result the operations procedure is presented (fig. 1, d) which needs to be considered for originally noncomplete data set $\left(N \neq 2^{m}\right)$. The functional properties of wavelet coefficients are considered in detail in paper [1].

\section{Description Algorithm of the Graph Similar to Two-Peak Characteristics with Features of Wavelet Coefficients}

In practice task it is very often to cut off the peak moments for calculations simplification, because they have small duration. However these values need the most careful control and research.

For example, the electricity tariff for the industrial enterprises depends of the extra consumption. In case of peaks smoothing of the measured signal, the Power Supply Company will have economic losses.

On the basis of the above properties, it was created the general algorithm is applicable for two-peak characteristics with keeping the main properties of a signal.

Let see in detail all stages of below presented algorithm. For better interpretation of the principle of the property definition for the offered algorithm, the approach of the original data depicting in wavelet coefficients with restriction by the quantity will be presented for the third level of wavelet decomposition (fig. 2).
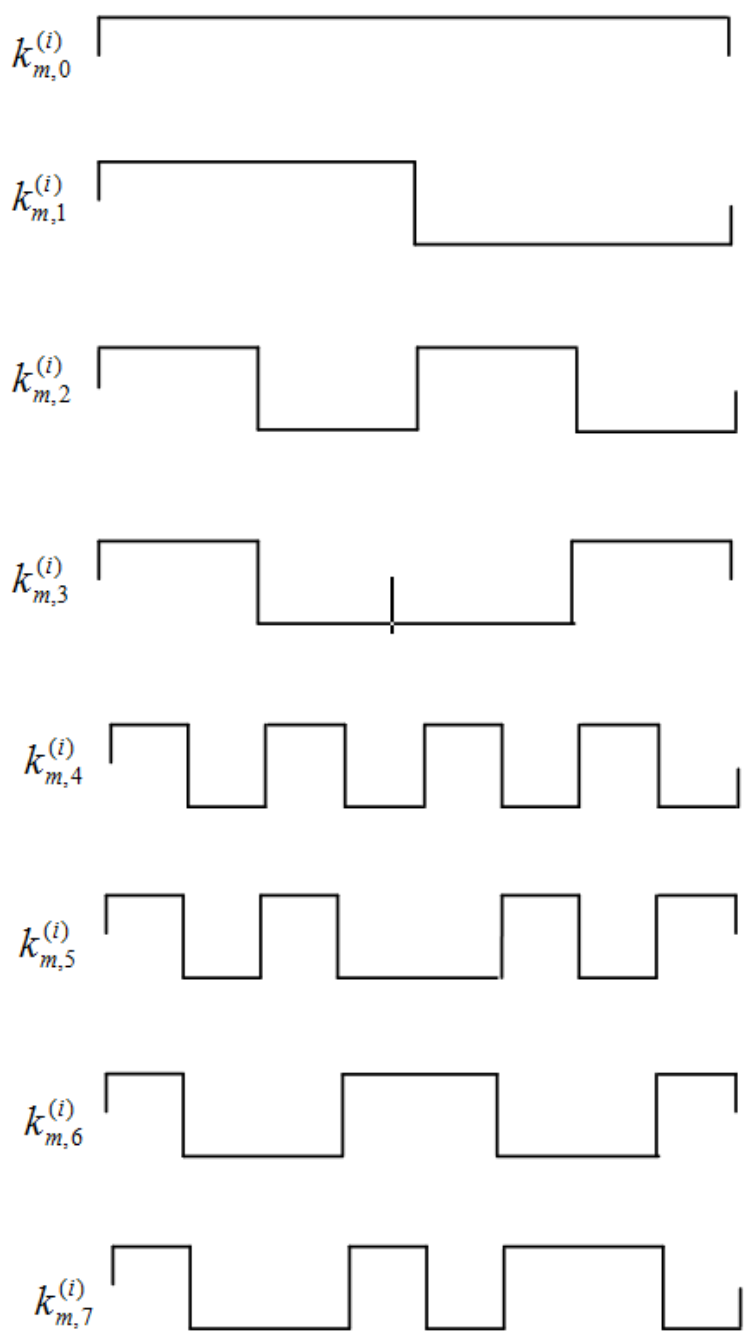

Fig. 2. The principle of the original signal depicting in wavelet coefficients (the quantity of wavelet coefficients in corresponds to the third level).

For daily graphs the segmentation has a large influence. The transition from the $(m-2)$ level of signal decomposition is presented in a matrix form:

$$
\left(\begin{array}{c}
k_{m, n} \\
k_{m, n+1} \\
k_{m, n+2} \\
k_{m, n+3}
\end{array}\right)=\mid \begin{array}{lcc}
k_{m-2, n}^{I} & k_{m-2, n}^{I I} & k_{m-2, n}^{I I I} \\
+ & + & + \\
+ & + & - \\
+ & - & + \\
+ & - & -
\end{array}
$$

\section{Data Recovery with Shifting in Wavelet Coefficients}

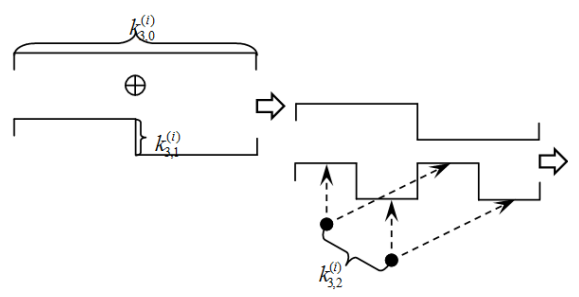

Fig. 3. Information about the original signal in wavelet coefficients. 
The developed approach is based on the following property. Wavelet coefficient is responsible for the value shifting to present a difference between half of values of the original signal (fig. 3). Graphical representation (fig. 3) also additional confirms the character of approximated and detailed wavelet coefficients. Especially, it is the property of information accumulation about the main part of a signal and its deviations respectively. Proceeding from fig. 3, the search algorithm can be presented on Table.

Table 1. Recovery algorithm of signal with functions shifting.

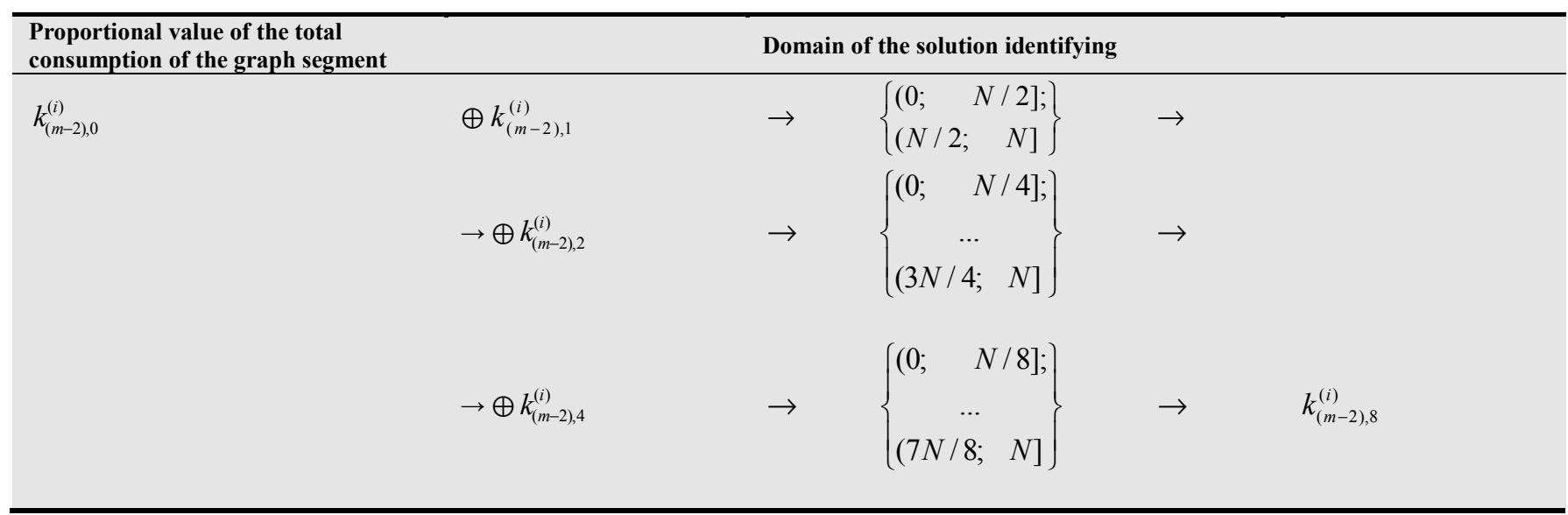

On the basis of tabular data presentation and sequence of data recovery, it is possible to make the next conclusion.

CONCLUSION 2: For determination of values in one segment of the original signal, it is necessary to take into account shifting of wavelet coefficients and to use only a half of the transformed values (fig. 2).

Initially, according to the MRA, the calculation of detailed wavelet coefficients is made for a possibility to detect fluctuations in the original signal. However, as a rule, it is widespread to use. Therefore the important properties of other detailed wavelet coefficients are lost which consider the following law of signal distribution (fig. 5). For simplicity, let us to consider the first stage of proposed algorithm (fig. 4 and Table).

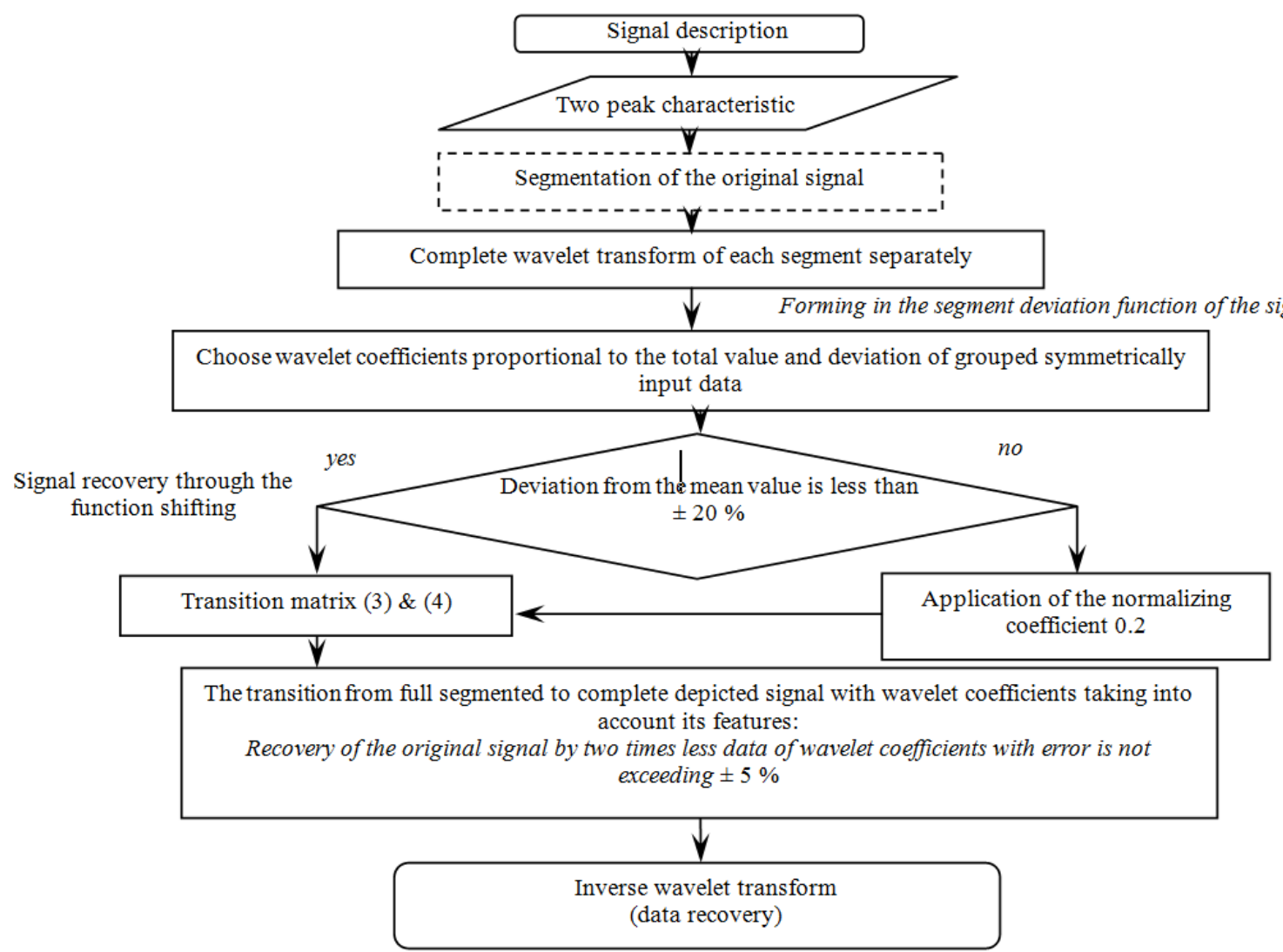

Fig. 4. Algorithm of the graph description similar to two-peak characteristic with features of wavelet coefficients. 


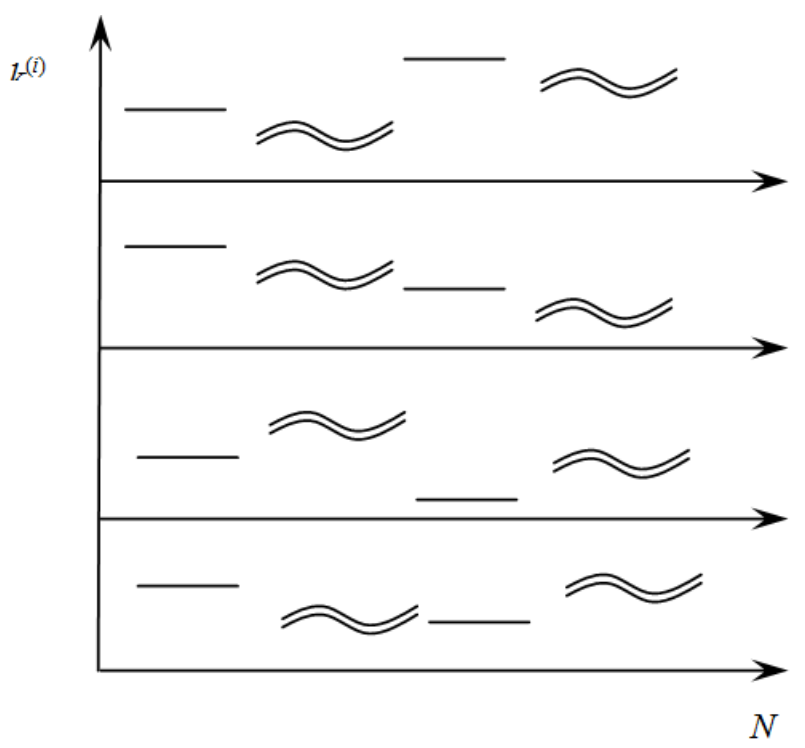

Fig. 5. Possible options of distribution of restored values with functions shifting.

Pair recovering of the summarized data of the original signal at present level of wavelet decomposition is carried out according to the equation:

$\left\{\begin{array}{ll}k_{(m-2), 2(1)}=\frac{k_{(m-2), 1(1)}+k_{(m-2), 2} ;}{2} & k_{(m-2), 2(2)}=\frac{k_{(m-2), 1(1)}-k_{(m-2), 2}}{2} \\ k_{(m-2), 2(3)}=\frac{k_{(m-2), 1(2)}+k_{(m-2), 2} ;}{2} & k_{(m-2), 2(4)}=\frac{k_{(m-2), 1(2)}-k_{(m-2), 2}}{2}\end{array}\right\}$

NOTE. Stage-by-stage transition between levels is provided with well-known equations (1) and (2).

CONCLUSIONS. As a research result, the description algorithm of nonlinear graphs is developed and offered which allows reducing twice the quantity of original data. However the error does not exceed normally acceptable limits which can be used in Power Engineering. Also in the paper, the scheme of properties of the Multiresolutional analysis is generalized that allows to present and to apply more effective detailed wavelet coefficients for creation new models and methods of signal processing.

\section{References}

[1] A. Voloshko, T. Lutchyn, "Principle of Determining Informative Wavelet Transformed Values in a Partial Restoration of Original Data Sample", Registration, Storing and Data Process, 14:4 (2012), 33-40. - (in Rus.).

[2] W. Lian, A. Mao, L. Zhang, "A Method for Cascading Failure Simulation based on Static Security Analysis", 10.1109/APPEEC.2009.4918053.

[3] A. Vahidnia, A. Dastfan, M. Banejad, "Determination of Harmonic Load Characteristics in Distribution Networks of Cities" Power Engineering, Energy and Electrical Drives, 2009. POWERENG '09. Intern. Conf., (2009), 442-446.
[4] Z. Zakaria, K. L. Lo, "Two-stage Fuzzy Clustering Approach for Load Profiling", Universities Power Engineering Conference (UPEC), 2009 Proc. of the $44^{\text {th }}$ Intern., (2009), 1-5.

[5] X. Ge, L. Zhang, Ya. Yang, "Multi-objective Hydro Optimal Scheduling with Flow Time", 10.1109/PowerCon.2012.6401259.

[6] L. Kipinski, R. Konig, C. Sieluzycki, “Application of modern tests for stationarity to singletrial MEG data. Transferring powerful statistical tools from econometrics to neuroscience", Biological Cybernetics, 105 (2011), 183-195.

[7] Yu. Yagi, Yu. Fukahata, "Introduction of uncertainty of Green's function into waveform inversion for seismic source processes", Geophysical Journal International, 186 (2011), 711-720.

[8] A. C. Iliopoulos, G. P. Pavlos, "Global Low Dimensional Seismic Chaos in the Hellenic Region", International Journal of Bifurcation and Chaos, 20:7 (2010), 2071-2095.

[9] C. L. Bain, J. de Paz, J. Kramer, "Detecting the ITCZ in Instantaneous Satellite Data using Spatiotemporal Statistical Modeling: ITCZ Climatology in the East Pacific", American Meteorological Society, 24 (2011), 216-230.

[10] T. Kozu, K. Masuzawa, T. Shimomai, "Estimation of No* for the Two-Scale Gamma Raindrop Size Distribution Model and Its Statistical Properties at Several Locations in Asia", Journal of Applied Metrology and Climatology, 49 (2010), 676-686.

[11] F. Qi, X. Liu, Yi. Ma, "Synthesis of neural tree models by improved breeder genetic programming", Neural Computing \& Applications, 21 (2012), 515-521.

[12] M. M. Katsova, I. M. Livshits, J. Sykora, "The Rotation of the Sun as a Star from the Green-Line Emission of the Entire Corona", Astronomy Reports 86:4 (2009), 343-354.

[13] Th. Candela, F. ois Renard, M. Bouchon, "Characterization of Fault Roughness at Various Scales: Implications of ThreeDimensional High Resolution Topography Measurements", Pure \& Applied Geophysic, 166 (2009), 1817-1851.

[14] R. Liu, M. Chen, H.-X. Wang, "Study on Grouping Control Strategy of AGC Units", 10.1109/ISGT-Asia.2012.6303348.

[15] J. A. Jardini, C. M. V. Tahan, E. L. Ferrari, S. U. Ahn, "Selection of Distribution Transformer Based on Economic Criteria", Electricity Distribution. Part 1: Contributions. CIRED. $14^{\text {th }}$ Intern. Conf. and Exhibition, 6 (1997), 7.

[16] M. H. Shariatkhah, M. R. Haghifam, "Determining of Annual Distribution Feeder Configuration Using Load Curves Clustering", Electrical Power Distribution Networks (EPDC), $201116^{\text {th }}$ Conf., (2011)

[17] Ch. Preet, G. Yadwinder, B. Kanwardeep, "Incentive Based Demand Response Program: an Effective Way to Tackle Peaking Electricity Crisis", 10.1109/CCECE.2012.6334813.

[18] G. I. Rhodes, "A Method of Studying Power Costs with Reference to the Load Curve and Overload Economies" American Institute of Electrical Engineers, (1912), 81-100.

[19] S. Kahrobaee, R. A. Rajabzadeh, L-K. Soh, "A Multiagent Modeling and Investigation of Smart Homes With Power Generation, Storage, and Trading Features", Smart Grid, IEEE Transactions, 4:2 (2012), 659-668. 
[20] S. V. Verdu, M. O. Garcia, F. J. G. Franco, "Characterization and Identification of Electrical Customers Through the Use of Self-Organizing Maps and Daily Load Parameters", Power Systems Conf. and Exposition, 2004. IEEE PES, 2 (2004), 899-906.

[21] J. A. Jardini, C. M. V. Tahan, M. R. Gouvea, "Daily Load Profiles for Residential, Commercial and Industrial Low Voltage Consumers", Power Delivery, IEEE Transactions, 15:1 (2000), 375-380.

[22] A. L. Shenkman, "Energy Loss Computation by Using Statistical Techniques", IEEE Transactions on Power Delivery, 5:1 (1990), 254-258.

[23] P. Kadar, "Understanding customer behavior", 10.1109/TDCLA.2008.4641800.

[24] Sh. Yang, Yi. Zhang, "Short-term Load Forecast Based on Decomposition of Daily Load Curve", 2010 International Conference on Computer, Mechatronics, Control and Electronic Engineering (CMCE) 3 (2010), 65-68.

[25] Yu. Sabri, N. Hariyanto, F. Fitriana, "Spatial Short-Term Load Forecasting using Grey Dynamic Model Specific in Tropical Area", 10.1109/ICEEI.2011.6021776.

[26] Q. Ding, J. Lu, H. Liao, “A Practical Super Short Term Load Forecast Method and Its Implementations", Power Systems Conference and Exposition, 2004. IEEE PES, 1 (2004), 483486 .

[27] M. B. Tasre, V. N. Ghate, P. P. Bedekar, "Comparative Analysis of Hourly Load Forecast for a Small Load Area", 2012 International Conference on Computing, Electronics and Electrical Technologies (ICCEET) (2012), 80-85.

[28] F. X. Xie, M. Huang, W. G. Zhang, "Research on Electric Vehicle Charging Station Load Forecasting", The International Conference on Advanced Power System Automation and Protection 2011 IEEE (2011), 2055-2060.

[29] R. Afkhami, F. M. Yazdi, "Application of Neural Networks for Short-Term Load Forecasting", 10.1109/POWERI.2006.1632536.

[30] M. Lopez, S. Valero, C. Senabre, “A SOM Neural Network Approach to Load Forecasting. Meteorological and Time Frame Influence", 10.1109/PowerEng.2011.6036553.

[31] J. Nazarko, A. Jurczuk, W. Zalewski, "ARIMA Models in Load Modelling with Clustering Approach", 10.1109/PTC.2005.4524719.

[32] P. T. T. Binh, N. T. Hung, P. Q. Dung, "Load Forecasting Based on Wavelet Transform and Fuzzy Logic", 10.1109/PowerCon.2012.6401281.
[33] I. Erkmen, A. Ozdogan, "Short Term Load Forecasting Using Genetically Optimized Neural network Cascaded with a Modified Kohonen Clustering Process", Proceedings of the $12^{\text {th }}$ IEEE International Symposium on Intelligent Control (1997), 107-112.

[34] D. Srinivasan, A.C. Liew, C.S. Chang, "Forecasting daily load curves using a hybrid fuzzyneutral approach", IEE Processing-Generation, Transmission, Distribution, 141:6 (1994), 561-567.

[35] D. M. FalcZio, H.O. Henriques, "Load Estimation in Radar Distribution Systems Using Networks and Fuzzy Set Techniques", Power Engineering Society Summer Meeting, 2 (2001), 1002-1006.

[36] M. Elsayed, "An overview of wavelet analysis and its application to ocean wind waves", Journal of Coastal Research, 26:3 (2010), 535-540.

[37] G. M. Menanno, A. Mazzotti, "Deconvolution of multicomponent seismic data by means of quaternions: Theory and preliminary results", Geophysical Prospecting, 60 (2012), 217-238.

[38] M. R. Homaeinezhad, M. Aghaee, H. N. Toosi, “Application of the Discrete Wavelet Transform for the Robust Detection of the Impulsive Incidences: Application to Arterial Blood Pressure Characteristic Events Detection-Delineation", International Journal of Wavelets, Multiresolution and Information Processing, 9:5 (2011), 813-842.

[39] G. Hall, S. Woodborne, M. Pienaar, "Rainfall control of the $\delta 13 \mathrm{C}$ ratios of Mimusops caffra from KwaZulu-Natal, South Africa", The Holocene, 19:2 (2009), 251-260.

[40] Kh. Mistry, R. Roy, "Enhancement of Voltage Stability Index of Distribution System by Network Reconfiguration Including Static Load Model and Daily Load Curve", Innovative Smart Grid Technologies - India (ISGT India), 2011 IEEE PES21 (2011), 65-68.

[41] D. Raisz, A. M. Dan, "Ripple Control as a Possible Tool for Daily Load Balancing in an Open Electricity Market Environment", 10.1109/PTC.2005.4524451.

[42] I. M. Dremin, O. V. Ivanov, V. A. Nechytailo, "Wavelets and its Usage”, SFS, 171:5 (2001), 465-501. - (in Rus.).

[43] A. Voloshko, T. Lutchyn, "Analysis of the Efficiency of Measures Compress of Schedules of Electrical Loads Аналіз ефективності міри стиснення графіків електричних навантажень", Vinnitsa NTU, 16:4 (2011), 209-214. - (in Ukr.). 